\title{
Variations
}

Variations

Revue internationale de théorie critique

$8 \mid 2006$

Subjectivités libres et critique de la répression

\section{Pour une écoute sociologique}

Répression, inhibition, prise de parole

\section{Alexander Neumann}

\section{(2) OpenEdition}

Journals

Édition électronique

URL : http://journals.openedition.org/variations/501

DOI : 10.4000/variations.501

ISSN : 1968-3960

Éditeur

Les amis de Variations

Édition imprimée

Date de publication : 1 novembre 2006

Référence électronique

Alexander Neumann, «Pour une écoute sociologique», Variations [En ligne], 8 | 2006, mis en ligne le 27 décembre 2012, consulté le 20 avril 2019. URL : http://journals.openedition.org/variations/501 ; DOI : 10.4000/variations.501

Ce document a été généré automatiquement le 20 avril 2019.

Les ami•e•s de Variations 


\title{
Pour une écoute sociologique
}

\author{
Répression, inhibition, prise de parole
}

\author{
Alexander Neumann
}

\section{NOTE DE L'AUTEUR}

Le présent article s'appuie sur un chapitre de ma thèse de sociologie, Les 35 heures flexibles. Le salariat entre sphère privée, entreprise et espace public, soutenue en 2005 à la SorbonneParis 5 et qui a obtenu les félicitations unanimes du jury.

1 La lente poussée sécuritaire du moment assoit son principe répressif sur une apparente atonie des citoyens, alors que ses instigateurs prétendent que les acteurs de la contestation ne porteraient pas de discours politique, justifiant ainsi leur marginalisation au sein du jeu de la représentation démocratique. Banlieusards, étrangers et sans papiers, lycéens, femmes révoltées, ouvriers en grève, petits paysans, se voient traités comme des objets apolitiques, car dénués d'une représentation reconnue comme étant légitime du point de vue de l'État républicain. La conséquence logique est leur criminalisation, leur mise au ban de la loi. L'absence d'une prise de parole de ces acteurs qui serait conforme aux codes de l'espace public bourgeois ${ }^{1}$ autorise ceux qui en contrôlent l'accès à proclamer l'inexistence d'une quelconque parole autonome de ces sujets-objets.

2 Simultanément, la plupart des théories sociologiques contemporaines suggèrent que les salariés, tout comme l'ensemble des acteurs dits subalternes, dominés et soumis, sont incapables de prendre la parole. Dans sa conceptualisation la plus formalisée, Pierre Bourdieu a jugé que la domination symbolique, exercée par la société bourgeoise, empêcherait tout simplement les ouvriers de s'exprimer publiquement ${ }^{2}$. D'une manière paradoxale, cette théorie rejoint l'argument de Jürgen Habermas selon lequel le monde du travail serait soumis à une socialisation marchande et bureaucratique qui interdirait de penser la prise de parole des salariés. À un moment donné, Habermas imagine même que les « variations plébéiennes » de l'espace public auraient été définitivement écrasées par la modernité capitaliste ${ }^{3}$. Les deux auteurs ont par la suite coupé ce vin trop sec par une larme d'eau, le premier en examinant les formes d'expression de la «misère du 
monde ", le second en avouant que les publics plébéiens se sont, jusqu'à ce jour, heurtés à la forme bourgeoise dominante de l'espace public ${ }^{4}$. Il n'en reste pas moins que leurs œuvres majeures évoquent des salariés sans subjectivité propre, sans capacités expressives et dénués de toute parole politique. Alain Touraine imagine à son tour que ces acteurs ne peuvent pas communiquer à travers un espace public, ce qui revient à penser qu'ils ne peuvent pas verbaliser leur expérience sociale ${ }^{5}$. L'arrière-fond de ces interprétations se trame dans la critique radicale qu'Hannah Arendt adresse au mouvement ouvrier européen, à la suite de l'écrasement stalinien de la révolution des conseils hongroise de $1953^{6}$. La philosophe considère alors que les organisations de masse issues d'un mouvement historique, mais largement bureaucratisées, sont réduites à des instances apolitiques, favorisant l'apologie d'un travail productif sans action, et d'une vie privée sans création. À la différence de Touraine et d'Habermas, Arendt n'affirme nulle part que les ouvriers ne puissent pas agir et communiquer à travers l'espace public, mais elle regrette plutôt que le travail aliéné et les organisations de masse entravent leurs capacités discursives. Pourquoi les acteurs de la société qui vivent en dehors de la culture bourgeoise dominante ont-ils du mal à prendre la parole? Telle est la question que nous avons envie de poser en tant que chercheur, au travers un déplacement de terrain. Le recours à la Théorie critique ouvre quelques pistes prometteuses. Elles passent par la rencontre de Freud, Kracauer, Negt, Adorno, et par quelques références à la sociologie hexagonale, afin d'arriver à une certaine écoute.

\section{Peur, inhibition, dépassement}

Dans son ouvrage Inhibition, symptôme et peur, Freud avance une relation possible entre la peur, la souffrance et la difficulté d'une prise de parole libre, et qui peut stimuler la réflexion sociologique actuelle ${ }^{7}$. Il définit alors la peur comme « une réaction à la menace de perdre l'objet d'identification». L'explication psychologique de la peur semble jusqu'ici concorder avec les interprétations sociologiques du rôle que joue le chômage, c'est-à-dire la menace d'une perte de l'emploi et du rôle social qui y correspond. Freud poursuit son argumentation en explorant les réactions alternatives à cette menace, dont la principale est la prise en compte de la nouvelle situation par le « travail de deuil ». Loin de concerner uniquement la mort d'une personne proche, le travail de deuil concerne toute situation de rupture, de perte d'une relation établie, et se distingue par la souffrance vive qu'il provoque. La peur apparaît ainsi comme une réaction plus rassurante que le deuil, qui permet seul le dépassement de la perte.

4 La peur joue ainsi comme un «mécanisme défensif » face à une situation qui pourrait s'avérer insupportable ou très douloureuse ${ }^{8}$. On peut en déduire que la peur et l'atonie sont des réactions convergentes, puisque le fait de nommer la perte, la menace de perte, ou une situation éprouvante, risquerait d'entraîner un travail de deuil et une souffrance aiguë.

5 Au travers de son travail sociologique novateur concernant la formation syndicale et ouvrière, Negt a transposé cette conception en mettant en relation l'expérience des restructurations des entreprises, la peur qu'elle provoque parmi les salariés, et les comportements conformistes, rassurants, que cela suscite. Il nous invite à écarter «le préjugé selon lequel l'activité psychique ne disposerait pas de la même matérialité que la réalité sociale manifeste ${ }^{9} »$. 
6 Rainer Zoll a pris au sérieux cette proposition, en menant une enquête intitulée «La conscience ouvrière en temps de crise ». L'auteur précise le rapport entre les réactions à la crise, la peur et les inclinations vers des attitudes conformistes ${ }^{10}$. Les entretiens menés par Zoll révèlent que, dans ces moments déstabilisants, les critiques radicales de l'entreprise et du capitalisme sont souvent repoussées par les salariés eux-mêmes, car elles soulignent l'ampleur du basculement qui est en train de se produire. Les militants syndicaux de gauche sont même taxés de "gauchistes délirants " par nombre de salariés. En réaction à cette stigmatisation, les militants se montrent à leur tour vexés et répliquent par d'autres invectives. La communication horizontale est neutralisée au fur et à mesure que l'identification directe avec l'entreprise s'accroît. Le sociologue allemand conclut:

Le ressort transformateur du schéma d'interprétation "contradiction capitaltravail" se brise donc contre son manque de concrétisation au sein de la vie quotidienne. [...] Dans la résignation, cette interprétation stagne et se ferme à de nouvelles expériences ${ }^{11}$.

7 L'explication sociologique s'inspire ici explicitement de la notion freudienne de « défense psychique ».

8 Le lien entre travail, peur et souffrance est également thématisé par Dejours ${ }^{12}$. Il mobilise non seulement l'expérience sociologique, mais aussi psychologique et clinique pour rendre compte des problèmes de stress, de harcèlement moral et de la question des réactions collectives à la réorganisation du travail. L'auteur constate que les réponses collectives des salariés d'un bureau, d'un atelier ou d'un site de production entier, face aux exigences du management ou de l'encadrement, prennent le plus souvent une forme informelle qui n'est pas toujours revendiquée publiquement. Il nomme ces réactions, qui ne sont pas immédiatement visibles, "stratégies défensives ${ }^{13}$ ", sans pour autant signaler clairement en quoi ce concept se distingue des « mécanismes défensifs » décrits par Freud 14 .

9 Comme ces phénomènes sont une partie intégrante de la modernité, il s'agit de développer une écoute sociologique appropriée. Autrement dit, il s'agit d'interroger, sinon de mettre en question, un certain genre de formalisation académique, qui tend à supprimer la particularité et la singularité de l'expression subjective.

10 L'un des exemples célèbres de la transgression des règles académiques formelles est l'enquête sociologique de Kracauer au sujet des Employés ${ }^{15}$ qui date de 1929: «Il a dialogué avec les uns et les autres, posant à chaque fois des questions différentes, menant ainsi son enquête tous azimuts ${ }^{16}$.» Le livre débute par la restitution d'un dialogue avec une jeune femme, rencontrée au hasard dans un train, le dimanche, et qui a visiblement « un petit coup dans le nez ». Même si la pertinence critique de cette étude a été saluée par les principaux représentants de l'École de Francfort, Benjamin constate que Kracauer a volontairement "laissé son chapeau de docteur en sociologie à la maison ». Loin de désavouer cette démarche hétérodoxe, il y voit un travail de conceptualisation supérieur à l'empirisme habituel : il s'agit d'une «tendre empirie qui se rend intimement identique à la chose et devient par là même véritablement théorie ${ }^{17} »$.

11 La différence avec une observation participante est que Kracauer ne suit aucune méthode formelle pour pénétrer l'univers du groupe social qui l'intéresse. L'exemple des employés esquisse l'un des deux points limites de la démarche sociologique : celui de l'objectivation maximale du discours des dominés, pratiquée par Bourdieu, et celui d'une compréhension de la subjectivité des acteurs, qui correspond à la démarche de Kracauer. Est-ce que le 
caractère contingent des contacts et l'état d'ébriété des personnes interrogées doivent être jugés comme une entorse méthodologique ou comme une garantie de la véracité du discours? Le dialogue de Kracauer avec la jeune femme, dont il a été question plus haut, montre une situation marquée par l'anonymat parfait (le train) et à un moment où les inhibitions sociales de la jeune femme semblent largement levées («le coup dans le nez »). Si le chercheur l'avait rencontrée dans le cadre de l'entreprise où elle travaille, il n'aurait probablement rien appris sur la double vie dont elle témoigne, entre ses aventures ludiques avec son patron et sa vie de couple modeste. Ce récit sur la double vie livre pourtant des renseignements précieux sur l'apparence sociale, qui est l'une des dimensions constitutives de la vie des employés allemands à cette époque.

De même, Boltanski rencontre des cadres dans un contexte privé, empli de connotations personnelles (liens d'amitié) qui assure un cadre de confiance facilitant la parole libre, pendant que plusieurs bouteilles de vin accompagnent l'enregistrement des récits. Où se situe alors la frontière entre l'entretien, le dialogue et la discussion, qui est censée définir la distance formelle du travail sociologique ? Boltanski a mis en garde contre une vision faussement objective des conditions de la prise de parole, par exemple quand il désigne l'entreprise comme un cadre qui inhibe l'expression des salariés en situation d'entretien :

La sociologie des entreprises qui a pour objet les lieux ou la domination de classe et la lutte entre les classes prennent les formes les plus intenses, les moins dissimulées ou les moins dissimulables est, plus que tout autre, tributaire de ses méthodes d'investigation et, très concrètement, des médiations par lesquels le terrain est approché18.

\section{Les barrières du langage}

13 Le caractère privé de l'entreprise ne saurait pourtant expliquer, à lui tout seul, les difficultés que rencontrent ouvriers et employés faiblement diplômés ou cultivés lorsqu'ils (elles) veulent prendre la parole. Cette difficulté surgit notamment lorsque ces acteurs sont obligés de s'exprimer dans un langage exigeant d'eux une cohérence formelle et logique. Lorsqu'ils s'apprêtent à verbaliser une situation de conflit ou des connaissances pratiques, lorsqu'ils tâchent d'exposer leurs attentes, les contraintes de l'expression lettrée entravent un langage qui prend appui sur les gestes du corps, les réactions immédiates de l'interlocuteur et la référence à des objets concrets ou aux particularités de la situation.

14 Negt a décliné cette problématique globale à partir de la prise de parole des ouvriers. Il constate :

Dans le mode d'expression des ouvriers, la relation la plus directe entre deux expériences différentes ne passe pas par une logique pure. L'ouvrier dépeint un bout d'une situation vécue au passage, pendant qu'il parle, tout en prenant une position en partie affective. [...] Au sein de l'espace public bourgeois, cet effort d'orientation est stigmatisé comme un détour inutile, notamment à l'école et à la télévision ${ }^{19}$.

Dans ce contexte, Negt parle d'une « barrière langagière » pour rendre visible la barricade formelle érigée par le langage codé d'une réunion officielle :

On coupe littéralement l'herbe sous les pieds (d'un ouvrier) si on le prive de toute possibilité de construire ses phrases en lien avec des choses sensibles, une situation concrète ou un interlocuteur. Cela s'applique aux assemblées et réunions au sein de l'entreprise ou à des témoignages devant le tribunal, où il est forcé de parler d'une 
manière abstraite, alors qu'il maîtrise bien mieux la description cohérente des relations dont il a fait l'expérience ${ }^{20}$.

16 Il ne s'agit donc pas d'une incapacité objective des ouvriers (ou d'autres salariés, par exemple les catégories précaires et immigrées) à formuler un discours pertinent, mais d'une entrave de leurs capacités subjectives d'expression dans un cadre imposé. Dans certaines enquêtes de sociologie menées en France, le même obstacle apparaît dans le rapport à l'écriture formelle ${ }^{21}$.

17 Si on suit la perspective de Negt, qui part des expressions subjectives et entravées des acteurs, il semble possible de capter certaines attentes divergentes et certains souhaits de dépassement des salariés, à condition d'assurer des conditions d'entretien très ouvertes. À ce propos, Boltanski fait remarquer que dans le doute, il est plus prometteur de choisir l'expression libre, au détriment de la méthode formelle :

[Nos] entretiens ont été menés selon des modalités que la plupart des manuels de méthodologie auraient sans doute réprouvées [...] Je me méfiais, par-dessus tout, de l'interview en bonne et due forme, accomplie sur le lieu de travail, avec la bénédiction de l'entreprise et de ses dirigeants ${ }^{22}$.

18 L'auteur a choisi de passer par des contacts informels, par des amis interposés, afin d'approcher les salariés, les faisant parler lors de dîners bien arrosés. Negt théorise l'absence d'une délibération naturelle au sein de l'entreprise, en la définissant comme un " espace privé de production », par opposition à l'espace public ${ }^{23}$. L'entreprise connaît certes des lieux de discussion, pendant les pauses, à la cantine, dans les couloirs, devant le distributeur de café, à l'occasion de réunions syndicales, etc., mais on peut penser que ces lieux transgressent déjà le cadre formel de l'entreprise. En général, les représentations fétichistes et marchandes y pèsent sur la perception des salariés, et sur leur capacité à nommer leurs souhaits et projets d'une manière autonome. La pratique discursive des salariés est constamment déformée, filtrée, sinon manipulée par le management, tandis que les situations vécues ne leur permettent souvent pas de comprendre l'ensemble de relations abstraites qui constituent l'entreprise.

\section{Pierre Bourdieu entre engagement et atonie}

19 Selon Bourdieu, la méfiance des dominés à l'égard du langage formel et de l'ordre symbolique dominant, autrement dit envers l'espace public bourgeois, ne serait qu'une forme indirecte de profonde reconnaissance des dominants, "maitres de l'art de mettre des formes et de payer de mots ${ }^{24} »$. L'apparente distance des dominés ne produit aucune connaissance, mais serait elle-même un acte de "méconnaissance ", se réduisant ainsi à une forme particulière de la reproduction sociale, quand les dominés «se contentent de ce qui leur est octroyé, mesurent l'espérance à leurs chances, se définissent comme l'ordre établi les définit ${ }^{25}$. " Cette incorporation complète de la domination, qui forme le support du concept d'habitus, ne peut être ébranlée que par la critique savante du sociologue et du scientifique, selon la vision bourdieusienne. La perspective d'un dépassement de cette condition par les salariés concernés devient ici improbable.

Patrick Cingolani refuse ce verdict et met en cause la posture du sociologue comme interprète et porte-parole des expériences des dominés: "Discours d'objectivation du populaire, La Distinction est en même temps le discours de la liquidation du peuple comme subjectivitée $^{26}$."L'auteur affirme que ce manque de prise en compte des capacités subjectives des salariés signe la limite étroite de cette théorie de la domination: "Pierre 
Bourdieu ne permet pas de conceptualiser les formes de rupture, de communication et de formation que traversent les classes populaires ». En réalité, les ouvriers s'expriment bien, en dépassant les situations vécues, grâce une parole organisée selon leurs propres principes. Il existe d'autres formes de médiation symbolique que la reproduction des situations subies ou l'apprentissage de la rhétorique formelle. Negt voit dans les espaces publics oppositionnels les lieux privilégiés de ce type de médiation, autorisant des langages variés et particuliers.

Sur le plan des interventions publiques de Bourdieu, la question paraît d'ailleurs moins tranchée :

Comment créer les bases d'un nouvel internationalisme, au niveau intellectuel, syndical et populaire? [...] Les bases sociales de la réussite d'une telle mobilisation existent, [par] l'affaiblissement de la coupure entre les étudiants et les travailleurs manuels [et] des liens réels se sont créés à travers, par exemple, les fils d'ouvriers éduqués touchés par la crise ${ }^{27}$.

Selon la conceptualisation bourdieusienne originaire cependant, les «membres des classes dominées » tiennent en permanence des discours en contradiction avec euxmêmes, en contradiction avec "le sens de leur pratique", livrés à la reproduction dominante sans moyen d'accéder à la " langue politique ${ }^{28}$."

Cingolani prend appui sur l'exemple du mouvement des chômeurs et des précaires pour esquisser la médiation symbolique qui permet pourtant aux dominés d'échapper à la simple reproduction, à la méfiance et à l'atonie ${ }^{29}$. La domination symbolique se montre ainsi dépassable.

Dans le documentaire La Repris ${ }^{30}$, Jeanne, la jeune femme salariée qui y fait figure d'antihéroïne, désigne la limite existentielle qui lui interdit d'entrer dans l'atelier, après la grève générale, refusant de plonger dans l'univers de la soumission, ce «trou noir dégueulasse » comme elle dit. Rien ne peut la faire bouger, ni l'autorité symbolique et masculine des cadres cégétistes, ni le charme discret de la direction. Après avoir perçu la vie autrement, l'atelier apparait non pas seulement comme l'image d'un travail épuisant et peu gratifiant, mais il signifie surtout la perte du sens de soi-même, qui vient d'être conquis. Ainsi, l'expérience amène la jeune femme à la révolte et à la prise de parole. Le cas limite de la délibération est celui d'un suicide ostentatoire sur les lieux de travail qui déclenche une enquête permettant de délier les langues des collègues ${ }^{31}$. Nous savons aussi que les revendications salariales, en apparence d'ordre économique, ont souvent caché des luttes pour la dignité ouvrière ${ }^{32}$. De même, les cadres d'IBM ont récemment créé des groupes d'expression indépendants pour s'opposer aux techniques du néo-management ${ }^{33}$.

L'enquête de Boltanski au sujet des cadres indique pourtant que les catégories moins soumises à la domination sont autant concernées par la violence symbolique que les ouvriers, précaires et immigrés, et les femmes salariées, plus faiblement qualifiées que les hommes. Même les cadres, dont l'un des traits caractéristiques est pourtant l'éloquence, appuyée sur un niveau de certification très élevé, peinent à surmonter l'inhibition et les effets de la domination sur la prise de parole autonome. En analysant l'expérience récente des cadres français, Jean-Pierre Le Goff a même constaté une "destruction du langage » sous l'effet des discours managériaux, auquel un grand nombre de cadres doivent se conformer ${ }^{34}$. Le langage volontairement abstrait, véhiculé par les écoles de management et les manuels d'expert, fonctionne comme une machine à détourner le sens pratique et la communication informelle. Dans certains cas de figure, la langue managériale tend même à neutraliser «l'éloquence", que Le Goff présente comme l'un des principaux traits du 
métier de cadre. Plusieurs cadres du secteur énergétique que nous avons nous-même interrogés s'interdisent en effet de commenter ouvertement la gestion du temps de travail, par exemple un jeune ingénieur rhénan : "Ici, la question des 35 heures est devenue indicible. On ne peut pas en parler au sein de l'entreprise... " Le cadre de la délibération est borné par l'orientation stratégique de l'entreprise multinationale, par des accords sur la flexibilité du temps de travail imposés sans consultation, par une programmation des projets répondant à un plan quinquennal, par les critères de rentabilité, etc.

\section{Limites des statistiques, frontières du monde administré} à l'usage des statistiques, destinées à évaluer la dimension subjective du rapport des salariés au travail. Baudelot et Gollac ont, par exemple, fait le pari d'une définition quantifiable des motivations particulières des salariés, en affirmant avec conviction que :

L'enquête statistique est de très loin l'approche la plus appropriée pour embrasser dans sa totalité, et surtout dans son extrême diversité, la nature contradictoire des relations que les individus entretiennent avec leur travail ${ }^{35}$.

La position exactement opposée à cette quantification statistique des subjectivités des salariés est défendue par André Gorz, qui met en avant les entretiens ouverts et individuels ${ }^{36}$. La question est de savoir s'il convient d'analyser la diversité, donc les particularités des motivations subjectives des salariés, par l'outil des statistiques. La qualité de l'expérience subjective, dont l'expression est par définition personnelle, se voit ici transformée en quantification. Autrement dit, la richesse des particuliers se trouve formulée sur le mode d'une généralisation logique. La "totalité ", qui est mentionnée plus haut dans la citation de Baudelot et Gollac, se réduit ainsi à une totalité abstraite qui se voit dans l'impossibilité de tenir compte des attentes ou raisons contradictoires qui forment l'horizon d'action des salariés, expulsant du discours sociologique les ambivalences de leur positionnement. Baudelot et Gollac assument ce choix, qui se désintéresse du contenu particulier des énonciations: "Davantage encore que le contenu intrinsèque des déclarations, ce sont les variations entre des réponses socialement différenciées qui intéressent le sociologue ${ }^{37}$."

28 Cette démarche statistique est davantage tournée vers les variations structurelles des réponses que vers les conceptions des salariés. En définitive, nous retrouvons une méthode s'inscrivant dans une tradition positiviste de la sociologie française, à travers laquelle «le systématique absorbe le spontané » et par laquelle le général domine le particulier ${ }^{38}$. Adorno et Horkheimer ont insisté sur la prééminence du général sur le particulier dans les sociétés modernes ${ }^{39}$, dont l'une des illustrations est fournie par le fonctionnement des mass media.

Cela signifie pour notre argumentation que les évaluations macro-sociologiques tendent à reproduire les généralisations et réifications sociales sans prendre suffisamment en compte les expériences particulières des acteurs. Dans ce cadre de réflexion, les enquêtes quantitatives servent en priorité à mettre en relief les contradictions générales de la société et non à cerner les réalités subjectivités. Ces catégories générales concernent par exemple les «différences essentielles dans les opinions fondamentales chez des sujets désignés 
comme appartenant à la classe supérieure ou inférieure, selon les critères statistiques les plus grossiers $^{40}$.»

30 La question à laquelle l'étude de Baudelot et Gollac se voit confrontée est : comment entendent-ils quantifier le bonheur, la dignité humaine et leurs interprétations subjectives complexes, à moins de rabattre ces valeurs au stade primaire d'une simple opinion de sondage? Pour cerner les critères de jugement des salariés, les responsables de l'enquête leur proposent par exemple de choisir parmi une liste de dix qualités humaines souhaitables, qui sont esquissées par des notions très générales, telles «le dynamisme ", « la culture ", « l'intelligence », « la compétence », etc. ${ }^{41}$ Ces notions sont tellement floues qu'elles sont souvent interprétées de façon contraire par différents acteurs. Ainsi, le discours managérial au sujet de la «compétence » peut facilement être opposé aux qualités humaines que les salariés entendent par là, par exemple la fiabilité dans les relations de travail, alors que pour le management, la compétence signifie flexibilité marchande et instabilité sociale. Les critères statistiques retenus excluent donc, par avance, les jeux de connotations et les significations ambivalentes des mots, qui prennent sens dans leur contexte particulier. L'orientation positiviste de cette recherche se manifeste dans l'extériorité de la méthode à son sujet, se contentant de classifier selon des indices généraux, comme si le phénomène social du rapport au travail était une réalité naturelle, donnée par avance, qui ne serait pas passée par la soumission historique du salariat. Adorno aborde ce problème dans l'un de ses nombreux articles sociologiques :

La génération de Max Weber a rappelé à juste titre que tout idéal de compréhension était toujours partiel, dans la mesure où il éliminait ce qui contrarie l'identification de celui qui comprend, au sein de la sociétét ${ }^{42}$.

31 Le philosophe et sociologue francfortois suggère une critique fondamentale de l'emploi des statistiques en sciences sociales, sans pour autant rejeter l'outil en soi. Lors de la "querelle sur le positivisme ", il constate que la méthode objectivante et objectiviste des statistiques est singulièrement appropriée pour décrire les tendances fétichistes du monde administré qui marquent les mentalités sociales ${ }^{43}$. Ces procédures techniques de la sociologie répondent donc à une réalité coiffée par la loi des moyennes et des grands nombres, autour de laquelle les comportements s'orientent. Demiroviç résume : «La méthode est juste, en ce qu'elle correspond à une société fausse». Les statistiques contribuent ainsi à une définition exacte de la société comme fait accompli, mais ne font pas apparaître les potentialités subjectives de son dépassement. L'objectif de la sociologie critique, soutenu par Adorno, est de favoriser un discours public visant une socialisation et un mode de vie qui soit capable de se soustraire aux critères statistiques.

Nous avons introduit plus haut les thèses de Negt, qui conceptualise l'opposition entre, d'un côté, les subjectivités entravées des salariés et, de l'autre, les abstractions formelles qui bloquent leur expression. En ce qui concerne l'usage spécifique des statistiques que nous venons d'évoquer, le questionnaire apparaît comme un langage très formalisé, qui restreint par avance les possibilités d'expression.

Les contradictions conceptuelles et pratiques des enquêtes statistiques portant sur la subjectivité des salariés renvoient, au fond, à une contradiction réelle qui se joue entre le travail vivant et les abstractions réelles dans lesquelles il est inséré ${ }^{44}$. Or, ces abstractions ne deviennent visibles qu'à travers un travail conceptuel approprié, qui rend du même coup audible la part subjective des salariés et qui n'adhère jamais intégralement à l'échange marchand et bureaucratique. Dans ce sens, le travail théorique constitue une pratique particulière de la démystification sociale qui ne se limite pas à la dénonciation 
d'une «fausse conscience ». Pour Adorno, il ne s'agit pas de déchirer le voile des fausses apparences pour faire surgir une réalité nue, proche de la vérité, mais de construire un cadre de compréhension et un langage qui rende possible des pratiques émancipatrices. La théorie n'est pas un mode d'emploi, mais doit offrir une orientation qui ne soit pas soumise à la nécessité ou aux représentations fétichistes qui dominent la perception sociale.

Face aux discours philosophiques souvent très abstraits de ses collègues, dont il réprouve les versants idéalistes, spéculatifs et métaphysiques, Adorno constate cependant que le concept critique ne peut saisir l'expérience sociale dans son intégralité. La raison principale en est le double caractère de l'expérience, comme expérience sensible du monde et comme interprétation subjective, mais conceptuelle, de ce monde par les acteurs ${ }^{45}$.

Dans sa Dialectique négative, il insiste donc sur l'impossibilité d'identifier les objets de la connaissance d'une manière univoque, à moins d'annuler le rôle de la critique. L'expérience singulière cherche des concepts pour nommer le monde social et pour le soumettre à la critique, tandis que le concept ne tire sa force que des expériences particulières, à moins de se réduire à une métaphysique.

\section{L'expérience, le concept, l'émancipation}

Dans ce cadre, la recherche empirique est saluée comme un apport indispensable à la construction conceptuelle, qui ne doit cependant pas rester à un niveau abstrait. Elle permet de produire des connaissances nouvelles et partielles, à condition de réfléchir sur son propre statut, et de révéler les manipulations pratiques de la subjectivité des acteurs (par exemple en démontant les astuces de la propagande idéologique, en déconstruisant les processus psychiques à l'œuvre dans les relations sociales ou encore en faisant apparaitre les manipulations techniques des statistiques). Cette attention empirique n'annule pas le travail théorique, seul accès à une interprétation de la société prise comme une totalité :

La totalité sociale, dont dépend la facticité de tous les particuliers, ne peut être déduite de la quantité du matériel recueilli, aussi riche qu'il puisse être, pas plus que la théorie ne peut être extrapolée à partir de résultats empiriques ${ }^{46}$.

Les enquêtes sociologiques conduites par Adorno et Horkheimer se concentrent, par conséquent, sur le rapport des acteurs aux abstractions et représentations dominantes. Leur recherche sur La personnalité autoritaire, publiée en $1950^{47}$, analyse les dispositions personnelles des personnes interrogées à l'égard de la politique, de la morale publique et de la sexualité, du syndicalisme, du capitalisme, etc., afin de favoriser la compréhension de l'émergence de tendances fascistes et antisémites dans la société nord-américaine. Pour circonscrire les attitudes plus ou moins autonomes ou autoritaires, les chercheurs construisent un questionnaire d'un genre nouveau, qui contourne les identifications directes, mais qui demande une prise de position sur des détails anodins de la vie quotidienne, opérant ainsi un déplacement de terrain. Les groupes de propositions qui concernent un seul aspect analytique sont par ailleurs dispersés, les questions tombent dans le désordre. Les acquis de la théorie freudienne, des enquêtes qualitatives préliminaires et des entretiens individuels accompagnent la réalisation du questionnaire, les aident à construire cette méthode. Le questionnaire est retravaillé à trois reprises, au fur et à mesure que les connaissances avancent. Voici deux propositions issues de la 
version finale, et qui concernent les "tendances autoritaires agressives", définies par "l'attention portée sur des hommes qui transgressent des normes conventionnelles, pour pouvoir les juger, stigmatiser et rejeter»: "Celui qui montre de mauvaises manières et une mauvaise éducation ne doit pas espérer s'entendre avec des gens honnêtes "; "Si les gens parlaient moins et travaillaient plus, tout le monde pourrait aller mieux ${ }^{48}$."

Les collaborateurs scientifiques d'Adorno, dont notamment Erich Fromm, cherchent ainsi à évaluer le degré d'autonomie des personnes interrogées, non pas à définir des lois macro-sociologiques. Le matériel d'enquête est soumis à un important travail d'interprétation par l'équipe de recherche, avant d'être conceptualisé, au lieu d'être considéré comme une donnée positive. Le dépassement, l'autonomie, l'émancipation restent l'oeuvre des personnes interrogées et ne sauraient être proclamés par un rapport de recherche.

\section{Ouverture}

$39 \mathrm{Au}$ lieu de conclure, disons que tous les citoyens pensent et parlent, raison pour laquelle la Théorie critique n'a pas dit son dernier mot. La question sociologique n'est plus de savoir si des salariés et d'autres acteurs réputés subalternes peuvent prendre la parole, puisqu'ils et elles le font constamment sans qu'on le leur demande, à travers les mouvements sociaux de ces dernières années, mais de savoir si les sociologues sont capables de les écouter, au lieu de les réduire au silence à l'aide d'arguments d'autorité.

\section{NOTES}

1. Pour traduire correctement le concept allemand bürgerliche Öffentlichkeit qu'utilise Habermas.

2. Pierre Bourdieu, La Distinction, Minuit, 1979, p. 549.

3. Jürgen Habermas, Strukturwandel der Öffentlichkeit, Suhrkamp, 1963/1990.

4. Pierre Bourdieu (Dir.), La misère du monde, Seuil, 1993 ; Habermas, « Vorwort zur Auflage von $1990 »$, in : Strukturwandel der Öffentlichkeit, Suhrkamp, 1963/1990, p. 21.

5. Voir Alain Touraine, Sociologie de l'action, Seuil, (1965) 1999.

6. Hannah Arendt, La Condition de l'homme moderne. Vita activa, Calman-Lévy, (1954) 1961.

7. Sigmund Freud, Hemmung, Angst und Symptom, Fischer, 1992, p. 110.

8. Jean Laplanche, Vocabulaire de la psychanalyse, PUF, 2002, p. 234.

9. Oskar Negt, Soziologische Phantasie und exemplarisches Lernen, 1971, p. 33-34.

10. Rainer Zoll, Arbeiterbewusstsein in der Krise, Bund Verlag, 1984.

11. Op. cit., p. 219.

12. Christophe Dejours, Souffrance en France, Seuil, 1998.

13. Op. cit., p. 42.

14. Cet usage approximatif des concepts trouve une prolongation dans l'analogie absurde entre l'entreprise et le camp de concentration, que l'auteur défend ouvertement.

15. Siegfried Kracauer, Die Angestellten, Suhrkamp, 1971.

16. Antonia Birnbaum, «K... sociologue », in : Abensour/Muhlmann (dir.), Tumultes, Kimé, 2002, p. 399. 
17. Op. cit., p. 417.

18. Luc Boltanski, Les Cadres, Minuit, 1982, p. 8.

19. Oskar Negt, Alexander Kluge, Öffentlichkeit und Erfahrung, Suhrkamp, 1972, p. 87.

20. Ibid., p. 91.

21. Stéphane Beaud, Michel Pialoux, La Condition ouvrière, Fayard, 1998, p. 250.

22. Luc Boltanski, op. cit., pp. 8-9.

23. Negt/Kluge, Öffentlichkeit und Erfahrung, Suhrkamp, 1972.

24. Pierre Bourdieu, La Distinction, Minuit, 1979, p. 541.

25. Op. cit., p. 549.

26. Patrick Cingolani, La République, les sociologues et le politique, La Dispute, 2003, p. 83.

27. Pierre Bourdieu, «Pour un nouvel internationalisme » in : A. Neumann (dir.), Les perspectives de la protestation, Syllepse, 1998, p. 30.

28. Pierre Bourdieu, op. cit., p. 538.

29. Patrick Cingolani, op. cit., p. 95

30. La Reprise. La fin de la grève à l'usine des piles Wonder à St. Ouen en 1968, réalisé par Hervé Leroux. 1996.

31. Christophe Dejours, op. cit.

32. Claude Durand, Pierre Dubois, La Grève, Presses de la Fondation nationale de sciences politiques, 1975.

33. Klaus Pickshaus (dir.), Arbeiten ohne Ende?,VSA, 2001.

34. Jean-Pierre Le Goff, Les Illusions du management, La Découverte, 2000, p. 77.

35. Christian Baudelot, Michel Gollac, 2003, Travailler pour être heureux ? p. 15.

36. André Gorz, Misères du présent, richesse des possibles, Galilée, 1997, p. 103.

37. Christian Baudelot, Michel Gollac, op. cit., p. 53.

38. Patrick Cingolani, op. cit., p. 75 ; voir aussi Miguel Abensour, «Le choix du petit ", postface à Theodor W. Adorno, Minima Moralia, Payot, 2001, p. 276.

39. Theodor W. Adorno, Max Horkheimer, La Dialectique de la raison; Payot, 1993.

40. Theodor W. Adorno, Soziologische Exkurse, Europäische Verlagsanstalt, 1953, p. 22 (nous traduisons).

41. Christian Baudelot, Michel Gollac, op. cit., p. 334.

42. Theodor W. Adorno, Soziologische Exkurse, Europäische Verlagsanstalt, 1953

43. Alex Demirović, Der non-konformistische Intellektuelle, Suhrkamp, 1999, p. 751.

44. Jean-Marie Vincent, Critique du travail, PUF, 1987 ; Oskar Negt, Arbeit und menschliche Würde, Steidl, 2001.

45. Cette définition refuse de considérer l'expérience à travers le seul prisme d'une description empirique et formalisée, grâce à un retour critique sur la conceptualisation hégelienne : G.W.F. Hegel, Phänomenologie des Geistes, Reclam, 1807/1987, p. 75.

46. Theodor W. Adorno, Friedrich Pollock, «Gruppenexperiment », in : Frankfurter Beiträge zur Soziologie, Suhrkamp, 1955, p. 5 (nous traduisons).

47. Adorno et alii, Studien zum autoritären Charakter, Suhrkamp, 1995.

48. Op. cit., p. 82. 


\section{AUTEUR}

\section{ALEXANDER NEUMANN}

Chercheur associé au Gepecs 